\title{
Estimação Cega de Canal para Sistemas MC DS CDMA ZP Baseada nos Métodos das Potências e de Subespaço
}

\author{
Deolinda F. Cardoso, Fabian D. Backx, Raimundo Sampaio Neto
}

\begin{abstract}
Resumo-Este trabalho investiga o problema da estimação cega de canal em sistemas MC DS CDMA ZP (Multi Carrier Direct Sequence Code Division Multiple Access Zero Padded). Usando somente o código de espalhamento do usuário de interesse, o método proposto, baseado no método das potências, estima a resposta ao impulso do canal multipercurso a partir do sinal recebido. Análises de desempenho simulado da taxa de erro de bit e de erro médio quadrático demonstraram que para moderada razão sinal ruído o método apresentado tem desempenho comparável aos tradicionais métodos de SVD mas com menor complexidade computacional.
\end{abstract}

Palavras-Chave-MC DS CDMA ZP, Estimação Cega de Canal, Técnicas de Potência.

Abstract - This work investigates the problem of blind channel estimation in Zero Padded Multi Carrier Direct Sequence Code Division Multiple Access (MC DS CDMA ZP). Using only the spreading code of the user of interest, the proposed method, based on the power method, estimates the impulse response of the multipath channel from the received data sequence. Mean square error and bit error rate performance analysis of the estimators through computer simulations reveals that for moderate signal to noise ratio the presented method achieves performance comparable to existing SVD techniques but at a lower computational cost.

Keywords-MC DS CDMA ZP, Blind Channel Estimation, Power Techniques.

\section{INTRODUÇÃO}

Recentemente, sistemas de transmissão em blocos, sejam eles de portadora única (Single Carrier - SC) ou de múltiplas portadoras (Multicarier - MC), têm sido amplamente investigados no contexto de esquemas com múltiplos usuários [1]. Dentre os sistemas de transmissão em blocos de múltiplas portadoras para sistemas multiusuário, destacam-se aqueles que, de alguma forma, empregam a técnica de múltiplo acesso por divisão de código (Code Division Multiple Access CDMA), propostos em [2], [3].

Uma dessas combinações resultou no sistema MC DS CDMA (Multicarrier Direct Sequence CDMA), foco deste estudo, no qual os símbolos de dados são espalhados no tempo e os chips da assinatura são todos transmitidos em cada uma das subportadoras, por meio de um esquema OFDM (Orthogonal Frequency Division Multiplexing) [4]. Esse sistema permite transmissões livres de Interferência Entre Blocos (IEB) (por

Centro de Estudos em Telecomunicações (CETUC), Pontifícia Universidade Católica do Rio de Janeiro (PUC-Rio), Brasil, E-mails: deolinda@cetuc.pucrio.br, fdb@cetuc.puc-rio.br, raimundo@cetuc.puc-rio.br. Este trabalho foi, parcialmente, financiado pelo Centro de Análises de Sistemas Navais (CASNAV-MB) e Conselho Nacional de Pesquisa e Desenvolvimento (CNPq) meio da utilização de um intervalo de guarda) e robustez contra os efeitos de desvanecimento do canal.

No caso de detecção coerente dos dados transmitidos, faz-se necessário estimar, na estação receptora, o canal de comunicação (usualmente, sua resposta ao impulso). Um dos modos de realizar a estimação do canal é por meio do envio de símbolos piloto; outro modo é a estimação cega na qual é necessário um conhecimento mínimo acerca, por exemplo, das estatísticas do sinal transmitido.

O objetivo do trabalho é propor, para o sistema MC DS CDMA, um método de estimação cega de canal, explorando a ortogonalidade dos subespaços do sinal e do ruído. Em um primeiro momento, para cada usuário, uma estimativa da resposta ao impulso do canal é obtida através do método de decomposição por valor singular 'svd padrão'. Essa estimativa é comparada com outras estimativas obtidas por um método de 'svd alternativo' no qual utilizou-se a inversa da matriz autocorrelação elevada a certa potência para aproximar um produto de matrizes contendo unicamente vetores que formam uma base do subespaço do ruído, bem como operações de produto ponto-a-ponto para amenizar a complexidade computacional. Finalmente, um estimador da resposta ao impulso do canal é obtido, com menor complexidade computacional.

Estimadores cegos de canal empregando o método das potências foram propostos para sistemas CDMA em [5], [6]. Todavia, foi somente em [7], [8] que uma explicação formal justificando o uso desse método foi apresentada. Os mesmos trabalhos mostraram, também, que estimadores obtidos por meio do método das potências são, na verdade, uma aproximação dos estimadores obtidos por meio do método de subespaço.

Ademais, as técnicas de estimação cega baseadas em subespaço apresentam um desempenho bastante próximo das abordagens que minimizam o erro médio quadrático (MSE) sem a necessidade de seqüências de treinamento, por isso, foram escolhidas neste trabalho. Dessa forma, partindo dos trabalhos de Doukopoulos [7], [8] propostos para CDMA e do trabalho de Backx [9] para OFDM, este estudo estende a metodologia para a estimação cega no sistema MC DS CDMA e avalia o seu desempenho em canais seletivos em frequiência.

Este trabalho está organizado da seguinte forma: a Seção 2 descreve o modelo vetorial dos sinais; a Seção 3 apresenta as análises de estimação cega; a Seção 4 descreve os resultados experimentais; e finalmente na Seção 5 apresentamos as conclusões do trabalho.

Notação adotada: caracteres maiúsculos em negrito de- 
notam matrizes; caracteres minúsculos em negrito denotam vetores. Os operadores $(.)^{T},(.)^{H}$ e $(.)^{*}$ indicam transposto de uma matriz, hermitiano (transposto conjugado) de um vetor, e conjugado de um escalar complexo; $\|\mathbf{A}\|_{F}$ representa a norma de Frobenius da matriz $\mathbf{A}$, e $\mathbf{A}^{-1}$ representa a matriz inversa de $\mathbf{A}$.

\section{O Modelo Vetorial dos Sinais}

São consideradas transmissões síncronas em $M$ subportadoras, no enlace reverso, em uma célula com $K$ usuários ativos. As seqüências de espalhamento são códigos curtos de WalshHadamard de comprimento $N$; os símbolos transmitidos são oriundos de constelações BPSK.

No sistema MC DS CDMA inicialmente ocorre a conversão série-paralelo de cada símbolo de informação a ser transmitido que resulta na formação de blocos de comprimento $M$. A seguir, os símbolos em cada bloco são multiplicados pela mesma seqüência de espalhamento, com ganho de processamento $N$. O bloco transmitido pelo $k$-ésimo usuário na $j$-ésima transmissão de chip $(j=0,1, \ldots, N-1)$ do $i$ ésimo bloco, denotado por $\mathbf{b}_{k}(i)$, é representado por $\mathbf{b}_{k}(i) c_{k, j}$ onde $c_{k, j}$ é o $j$-ésimo chip da seqüência de espalhamento $\mathbf{c}_{k}=\left[c_{k, 0}, \ldots c_{k, N-1}\right]^{T}$, que satisfaz $\left\|\mathbf{c}_{k}\right\|=1$.

Antes da transmissão do bloco (chip-a-chip), é aplicada uma operação de transformada inversa de Fourier (IFFT) de $M$ pontos; a seguir, um intervalo de guarda do tipo ZP (ZeroPadding) [10] de comprimento $G$ (no mínimo igual a ordem do canal) é inserido, ao final de cada bloco para garantir a eliminação da IEB. Esse bloco OFDM resultante, de dimensão $P=M+G$, é, então, transmitido pelo canal multipercurso, modelado por uma matriz de convolução Toeplitz $P \times P$ triangular inferior, na qual a primeira coluna é a resposta ao impulso do canal, no tempo, estendida com zeros:

$$
\mathbf{h}_{k_{e s t}}=\left[\begin{array}{llllll}
h_{k_{0}} & \ldots & h_{k_{L-1}} & 0 & \ldots & 0
\end{array}\right]^{T}
$$

onde $L$ é o comprimento do canal.

No receptor o intervalo de guarda não é removido do bloco recebido e, então, é computada a operação da transformada direta de Fourier (FFT) de $P$ pontos.

Considerando os $K$ usuários ativos do sistema, o agregado dos sinais recebidos, correspondente à transmissão do $j$-ésimo chip do $i$-ésimo bloco de símbolos pode ser escrito como:

$$
\mathbf{x}(i ; j)=\sum_{k=1}^{K} \widetilde{\mathbf{H}}_{k} \mathbf{V b}_{k}(i) c_{k, j}+\mathbf{n}(i ; j)
$$

onde $\mathbf{V}=\mathbf{F}_{P, P} \mathbf{F}_{P, M}^{H}$ é uma matriz estruturada de dimensões $P \times M$; a matriz $\mathbf{F}_{P, P}$ implementa uma FFT de $P$ pontos e é normalizada de forma que $\mathbf{F}_{P, P}^{H} \mathbf{F}_{P, P}=\mathbf{F}_{P, P} \mathbf{F}_{P, P}^{H}=\mathbf{I}_{P, P}$ onde $\mathbf{I}_{P, P}$ denota a matriz identidade $P \times P$; e $\mathbf{F}_{P, M}$ é uma matriz $P \times M$ contendo as primeiras $M$ colunas da matriz $\mathbf{F}_{P, P} ; \mathbf{n}(i ; j)$ é um vetor de ruído gaussiano branco complexo com matriz covariância $\mathrm{E}\left[\mathbf{n}(i ; j) \mathbf{n}^{H}(i ; j)\right]=\sigma^{2} \mathbf{I}_{P}$. A matriz $\widetilde{\mathbf{H}}_{k}=\operatorname{diag}\left(\tilde{\mathbf{h}}_{k}\right)$ é diagonal $P \times P$ cujas entradas contém a resposta em frequiência do canal $\widetilde{\mathbf{h}}_{k}$, dada por:

$$
\widetilde{\mathbf{h}}_{k}=\sqrt{P} \mathbf{F}_{P, P} \mathbf{h}_{k_{\text {est }}} \text {. }
$$

Após coletar $N$ blocos de chips consecutivos é possível formar, para o $i$-ésimo bloco de símbolos, a matriz $P \times N$ :

$\mathbf{X}(i)=[\mathbf{x}(i, 0) \ldots, \mathbf{x}(i, N-1)]=\sum_{k=1}^{K} \widetilde{\mathbf{H}}_{k} \mathbf{V} \mathbf{b}_{k}(i) \mathbf{c}_{k}^{T}+\mathbf{N}(i)$

onde a matriz $\mathbf{N}(i)=[\mathbf{n}(i ; 0) \ldots, \mathbf{n}(i ; N-1)]$ contém os vetores de ruído presentes em cada transmissão de chip do $i$ ésimo bloco. Note que a resposta em frequiência do canal em (3) pode também ser expressa em função da resposta do canal no tempo, sem a extensão com zeros, de modo que:

$$
\widetilde{\mathbf{h}}_{k}=\sqrt{P} \mathbf{F}_{P, L} \mathbf{h}_{k},
$$

onde a matriz $\mathbf{F}_{P, L}$ tem dimensões $P \times L$ contendo as primeiras $L$ colunas da matriz $\mathbf{F}_{P, P}$. Neste estudo considerase que os comprimentos dos canais de cada usuário são todos iguais a $L$.

\section{EstimaÇÃo Cega de CANAl BASEAdA EM SubespaÇO E No MÉTOdo das PotênCIAS}

$\mathrm{Na}$ estação rádio base a matriz do agregado de sinais, equação (4), é processada por um banco de filtros casados às seqüências de espalhamento dos usuários ativos no sistema. $\mathrm{Na}$ saída do filtro casado ao código do $m$-ésimo usuário de interesse, não existe mais interferência de múltiplo acesso (IMA) (devido à preservação da ortogonalidade dos códigos empregados) e, assim, a matriz do agregado de sinais recebidos se reduz ao vetor do sinal, desespalhado, do $m$-ésimo usuário:

$$
\mathbf{y}_{m}(i)=\mathbf{X}(i) \mathbf{c}_{m}^{*}=\widetilde{\mathbf{H}}_{m} \mathbf{V} \mathbf{b}_{m}(i)+\mathbf{n}_{f}(i),
$$

onde $\mathbf{c}_{m}^{*}$ é o vetor conjugado do código de espalhamento do $m$-ésimo usuário; e $\mathbf{n}_{f}(i)=\mathbf{N}(i) \mathbf{c}_{m}^{*}$ é um vetor de ruído gaussiano branco complexo com matriz covariância dada por $\mathrm{E}\left[\mathbf{n}_{f}(i) \mathbf{n}_{f}^{H}(i)\right]=\sigma^{2} \mathbf{I}_{P, P}$, onde $\sigma^{2}$ é a potência do ruído.

A matriz autocorrelação do sinal desespalhado (6) é expressa por:

$$
\mathbf{R}_{\mathbf{y}_{m}}=\mathrm{E}\left[\mathbf{y}_{m}(i) \mathbf{y}_{m}^{H}(i)\right] .
$$

Empregando o método de decomposição por valor singular (Singular Value Decomposition SVD) [11] tem-se:

$$
\mathbf{R}_{\mathbf{y}_{m}}=\left[\mathbf{S}_{s} \mathbf{S}_{n}\right]\left[\begin{array}{cc}
\boldsymbol{\Lambda}_{s}+\sigma^{2} \mathbf{I}_{M} & \mathbf{0} \\
\mathbf{0} & \sigma^{2} \mathbf{I}_{G}
\end{array}\right]\left[\begin{array}{l}
\mathbf{S}_{s}^{H} \\
\mathbf{S}_{n}^{H}
\end{array}\right]
$$

onde $\mathbf{S}_{s}$ é uma matriz de dimensões $P \times M$ cujas colunas formam uma base ortonormal para o subespaço dos sinais, e a matriz $\mathbf{S}_{n}$ é uma matriz de dimensões $P \times G$ cujas colunas formam uma base ortonormal para o subespaço do ruído.

A matriz $\boldsymbol{\Lambda}_{s}$ é uma matriz diagonal contendo os $M$ valores singulares associados aos $M$ vetores singulares da matriz $\mathbf{S}_{s}$ do subespaço dos sinais, isso sugere que o subespaço dos sinais tem posto $M$ e, sendo o subespaço do ruído o seu complemento ortogonal, este tem posto $G$.

$\mathrm{Na}$ equação (6), as colunas da matriz $\widetilde{\mathbf{H}}_{m} \mathbf{V}$, de dimensões $P \times M$, estão contidas no subespaço dos sinais e, portanto, são ortogonais ao subespaço do ruído sendo válido aplicar as seguintes igualdades: 


$$
\mathbf{S}_{n}^{H} \widetilde{\mathbf{H}}_{m} \mathbf{V}=\mathbf{0}_{G, M} \Leftrightarrow\left\|\mathbf{S}_{n}^{H} \widetilde{\mathbf{H}}_{m} \mathbf{V}\right\|_{F}^{2}=0
$$

Assim, conforme descrito em [9], é possível chegar à seguinte expressão:

$$
\begin{aligned}
\left\|\mathbf{S}_{n}^{H} \widetilde{\mathbf{H}}_{m} \mathbf{V}\right\|_{F}^{2} & =\widetilde{\mathbf{h}}_{m}^{H}\left[\sum_{j=0}^{M-1} \operatorname{diag}\left(\mathbf{v}_{j}\right)^{H} \mathbf{S}_{n} \mathbf{S}_{n}^{H} \operatorname{diag}\left(\mathbf{v}_{j}\right)\right] \widetilde{\mathbf{h}}_{m}(10) \\
& =0,
\end{aligned}
$$

onde foi usado $\mathbf{V}=\left[\begin{array}{llll}\mathbf{v}_{0} & \mathbf{v}_{1} & \ldots & \mathbf{v}_{M-1}\end{array}\right]$.

Uma estimativa $\widehat{\widetilde{\mathbf{h}}}_{m}$ do vetor $\widetilde{\mathbf{h}}_{m}$ é o vetor singular associado ao menor valor singular (que é zero), da matriz dada por $\sum_{j=0}^{M-1} \operatorname{diag}\left(\mathbf{v}_{j}\right)^{H} \mathbf{S}_{n} \mathbf{S}_{n}^{H} \operatorname{diag}\left(\mathbf{v}_{j}\right)$ de dimensões $P \times P$. Convém frisar que essa estimativa é da forma $\widehat{\widetilde{\mathbf{h}}}_{m}=\beta \widetilde{\mathbf{h}}_{m}$ contendo, portanto, uma ambigüidade de fase modelada por um escalar complexo $\beta$.

\section{A. A idéia Chave}

Conforme visto, para obter uma estimativa $\widehat{\widetilde{\mathbf{h}}}_{m}$ da resposta em freqüência do canal é preciso determinar o vetor singular associado ao menor valor singular de uma matriz de dimensões $P \times P$, o que pode ser conseguido aplicando-se, por exemplo, uma SVD na matriz $\sum_{j=0}^{M-1} \operatorname{diag}\left(\mathbf{v}_{j}\right)^{H} \mathbf{S}_{n} \mathbf{S}_{n}^{H} \operatorname{diag}\left(\mathbf{v}_{j}\right)$.

Contudo, a seguir são explorados alguns fatos que permitem amenizar a complexidade computacional, bem como melhorar a qualidade do estimador proposto.

1) Aplicação de Produto Ponto-a-Ponto:

Na expressão $\sum_{j=0}^{M-1} \operatorname{diag}\left(\mathbf{v}_{j}\right)^{H} \mathbf{S}_{n} \mathbf{S}_{n}^{H} \operatorname{diag}\left(\mathbf{v}_{j}\right)$ é possível empregar as seguintes operações:

$$
\operatorname{diag}\left(\mathbf{v}_{j}\right)^{H} \mathbf{S}_{n} \mathbf{S}_{n}^{H} \operatorname{diag}\left(\mathbf{v}_{j}\right)=\left(\mathbf{v}_{j}^{*} \mathbf{v}_{j}^{T}\right) \odot \mathbf{S}_{n} \mathbf{S}_{n}^{H},
$$

e

$$
\left(\sum_{j=0}^{M-1} \mathbf{v}_{j}^{*} \mathbf{v}_{j}^{T}\right) \odot \mathbf{S}_{n} \mathbf{S}_{n}^{H}=\mathbf{V}^{*} \mathbf{V}^{T} \odot \mathbf{S}_{n} \mathbf{S}_{n}^{H} .
$$

Assim, os somatórios são substituídos por um produto ponto-a-ponto e a expressão (10) é simplificada a:

$\widetilde{\mathbf{h}}_{m}^{H}\left[\sum_{j=0}^{M-1} \mathbf{v}_{j}^{*} \mathbf{v}_{j}^{T} \odot \mathbf{S}_{n} \mathbf{S}_{n}^{H}\right] \widetilde{\mathbf{h}}_{m}=\widetilde{\mathbf{h}}_{m}^{H}\left[\mathbf{V}^{*} \mathbf{V}^{T} \odot \mathbf{S}_{n} \mathbf{S}_{n}^{H}\right] \widetilde{\mathbf{h}}_{m}$

2) Aproximação em Potência para a Matriz $\mathbf{S}_{n} \mathbf{S}_{n}^{H}$ :

Baseado no método das potências, o produto de matrizes $\mathbf{S}_{n} \mathbf{S}_{n}^{H}$ pode ser aproximado pela inversa da matriz autocorrelação elevada a uma potência $p$. Para isso partimos do seguinte Lema descrito por Doukopoulos em [12]:

Lema 1 - Seja a matriz autocorrelação $\mathbf{R}_{y_{m}}$ decomposta em SVD como em (8). Sejam $\lambda_{1} \geq \lambda_{2} \geq \ldots \lambda_{M} \geq 0$ os valores singulares e elementos componentes da diagonal da matriz $\Lambda_{s}$; assim podemos escrever:

$$
\left(\sigma^{2} \mathbf{R}_{\mathbf{y}_{m}}^{-1}\right)^{p}=\left[\mathbf{S}_{s} \mathbf{S}_{n}\right]\left[\begin{array}{cc}
\left(\frac{\boldsymbol{\Lambda}_{s}+\sigma^{2} \mathbf{I}_{M}}{\sigma^{2}}\right)^{-p} & \mathbf{0} \\
\mathbf{0} & \mathbf{I}_{G}
\end{array}\right]\left[\begin{array}{c}
\mathbf{S}_{s}^{H} \\
\mathbf{S}_{n}^{H}
\end{array}\right]
$$

A partir da expressão acima é possível observar que a matriz $\left(\frac{\boldsymbol{\Lambda}_{s}+\sigma^{2} \mathbf{I}_{M}}{\sigma^{2}}\right)^{-p}$ depende da potência $p$; desde que é uma matriz diagonal com elementos da forma $\frac{\lambda_{j}+\sigma^{2}}{\sigma^{2}}$ para $j=1, \ldots, M$ pode ser deduzido que:

$$
\lim _{p \rightarrow \infty}\left(\frac{\boldsymbol{\Lambda}_{s}+\sigma^{2} \mathbf{I}_{M}}{\sigma^{2}}\right)^{-p}=\mathbf{0}
$$

Finalmente, combinando as expressões (13) e (14) verificase a seguinte igualdade:

$$
\lim _{p \rightarrow \infty}\left(\sigma^{2} \mathbf{R}_{\mathbf{y}_{m}}^{-1}\right)^{p}=\mathbf{S}_{n} \mathbf{S}_{n}^{H}
$$

A expressão (15) é uma variação do método das potências.

Dessa forma, pode ser usada a matriz $\left(\mathbf{R}_{\mathbf{y}_{m}}\right)^{-p}$ para aproximar o produto $\mathbf{S}_{n} \mathbf{S}_{n}^{H}$. Quando a potência $p$ tende para o infinito, o resultado da aproximação converge para o produto $\mathbf{S}_{n} \mathbf{S}_{n}^{H}$, a menos de um escalar cujo valor não é necessário conhecer.

Sob as observações descritas, o vetor da estimativa da resposta em freqüência do canal pode ser obtido de forma a minimizar a forma quadrática dada por:

$$
\widehat{\widetilde{\mathbf{h}}}_{m}=\arg \min _{\|\widetilde{\mathbf{h}}\|^{2}=P}\left\{\widetilde{\mathbf{h}}^{H}\left[\mathbf{V}^{*} \mathbf{V}^{T} \odot \mathbf{R}_{\mathbf{y}_{m}}^{-p}\right] \widetilde{\mathbf{h}}\right\}
$$

com a restrição $\|\widetilde{\mathbf{h}}\|^{2}=P$ para evitar a solução trivial.

3) Obtenção da estimativa do canal no tempo.

É possível diminuir a complexidade do estimador dado em (16) e melhorar a sua qualidade. A partir de (5) sabemos que o vetor da resposta em freqüência do canal $\tilde{\mathbf{h}}_{m}$ pertence ao subespaço gerado pelas $L$ primeiras colunas da matriz FFT de $P$ pontos. Como o valor correto do comprimento $L$ do canal pode não ser conhecido a priori, assume-se o intervalo de guarda $G$, que é conhecido, como o comprimento do canal.

Reescrevendo a forma quadrática a ser minimizada em (16) no domínio do tempo, tem-se:

$$
\widehat{\mathbf{h}}_{m}=\arg \min _{\|\mathbf{h}\|=1}\left\{\mathbf{h}^{H} \mathbf{F}_{P, G}^{H}\left[\mathbf{V}^{*} \mathbf{V}^{T} \odot \mathbf{R}_{\mathbf{y}_{m}}^{-p}\right] \mathbf{F}_{P, G} \mathbf{h}\right\},
$$

e

$$
\widehat{\widetilde{\mathbf{h}}}_{m}=\sqrt{P} \mathbf{F}_{P, G}^{H} \widehat{\mathbf{h}}_{m},
$$

onde a restrição $\|\mathbf{h}\|=1$ em (17) evita a solução trivial.

Neste trabalho, a matriz autocorrelação é estimada como uma média temporal ao longo dos blocos de símbolos transmitidos:

$$
\widehat{\mathbf{R}}_{y_{m}}(i)=\frac{1}{i} \sum_{j=1}^{i} \mathbf{y}_{m}(j) \mathbf{y}_{m}^{H}(j) .
$$

Convém ressaltar que, sendo a matriz autocorrelação estimada, a condição de ortogonalidade $\left\|\mathbf{S}_{n}^{H} \widetilde{\mathbf{H}}_{m} \mathbf{V}\right\|^{2}=0$ em (9) não poderá ser sempre garantida. Em conseqüência da matriz ser estimada, o limite (15) ao invés de ser a matriz do produto desejado pode se tornar, apenas, uma matriz de posto 1 do tipo $\mathbf{s s}^{H}$ onde s é o vetor singular correspondente ao menor valor singular da matriz autocorrelação $\widehat{\mathbf{R}_{y_{m}}}(i)$ [12]. Esse fato faz com que nos estágios iniciais da estimação da matriz autocorrelação (poucos blocos de símbolos transmitidos) nem sempre o aumento da potência $p$ poderá garantir 
melhor desempenho. Entretanto, quando o número de blocos de símbolos transmitidos aumenta, a maior potência prevalece resultando em melhor desempenho; isto será verificado na seção de resultados experimentais.

A formulação adotada permite uma abordagem de menor complexidade computacional devido a não mais ser necessário computar somatórios e produtos de matrizes $\mathbf{S}_{n} \mathbf{S}_{n}^{H}$, especialmente quando essas matrizes e esses produtos têm de ser constantemente atualizados juntamente com as estimativas de $\mathbf{R}_{y_{m}}$. Os termos dos somatórios foram substituído por uma simples operação de produto ponto-a-ponto entre a matriz pré-computada $\mathbf{V}^{*} \mathbf{V}^{T}\left(\mathbf{V}=\mathbf{F}_{P, P} \mathbf{F}_{P, M}^{H}\right.$ envolve apenas matrizes de Fourier), e uma matriz de potência $\left(\widehat{\mathbf{R}}_{\mathbf{y}_{m}}^{-1}(i)\right)^{p}$ onde $\widehat{\mathbf{R}}_{\mathbf{y}_{m}}^{-1}(i)$ pode ser recursivamente calculada aplicando o Lema de inversão de matrizes.

Além disso, a obtenção da estimativa do canal no tempo dada em (17) consiste em determinar o vetor singular associado ao menor valor singular de uma matriz de dimensões $G \times G$, ou seja, de dimensões da ordem do canal.

Finalmente, é possível observar pela expressão dada em (14) que a velocidade de convergência, pelo uso da matriz de potência $\left(\widehat{\mathbf{R}}_{y_{m}}(i)\right)^{-p}$ para aproximar o produto $\mathbf{S}_{n} \mathbf{S}_{n}^{H}$, é exponencial; tendo o componente mais lento a seguinte forma $\left(\frac{\sigma^{2}}{\lambda_{j}+\sigma^{2}}\right)^{p}$, isso permite deduzir que essa aproximação é mais eficiente em ambientes com alta razão sinal ruído $E_{b} / N_{0}$. Em conseqüência, em tais ambientes, com pequenos valores da potência $p$ é possível aproximar de forma eficaz o produto $\mathbf{S}_{n} \mathbf{S}_{n}^{H}$ a partir das estimativas da matriz autocorrelação.

\section{B. Equalização e Detecção}

Após a estimação de canal é possível realizar a equalização de (6) seguida da detecção do símbolo; aplicando o equalizador ZF (zero-forcing), temos:

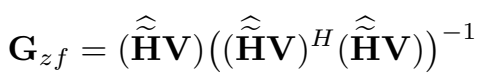

onde $\widehat{\widetilde{\mathbf{H}}}=\operatorname{diag}\left(\widehat{\widetilde{\mathbf{h}}}_{m}\right)$.

Outra possibilidade é aplicar o equalizador MMSE (Minimum Mean Square Error):

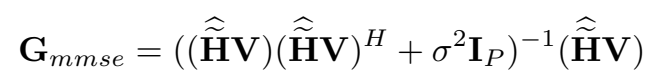

Após a equalização, uma estimativa do bloco transmitido $\mathbf{b}_{m}(i)$ pode ser obtida.

$$
\widehat{\mathbf{b}}_{m}(i)=\operatorname{sinal}\left(\operatorname{real}\left(\mathbf{G}^{H} \mathbf{y}_{m}(i)\right)\right)
$$

onde o operador sinal $(\operatorname{real}(\cdot))$ é aplicado a cada componente do vetor $\mathbf{G}^{H} \mathbf{y}_{m}(i)$ de dimensão $P \times 1$.

\section{RESUltados EXPERIMENTAis}

Nesta seção estão apresentados os resultados experimentais para o sistema MC DS CDMA com intervalo de guarda do tipo ZP. Os experimentos objetivam comparar o método de decomposição por valor singular SVD, descrito em [10], com a abordagem descrita neste estudo.

Dois tipos de canais foram usados nos experimentos: o primeiro tipo de canal é modelado como um filtro FIR de ordem 5. O comprimento do intervalo de guarda vale $G=8$. Uma vez que o intervalo de guarda é maior que a ordem do canal, fica garantido que não haverá interferência entre blocos na recepção. Os ganhos são normalizados de forma a $\|\mathbf{h}\|^{2}=1$ e o vetor de coeficientes da resposta ao impulso do canal simulado é dado por $\mathbf{h}=[0.74 ;-0.42 ; 0.083 ; 0.49 ;-0.12 ; 0.01]$. Conforme descrito em [13], esse canal apresenta um nulo espectral pronunciado, o que pode ser observado na Figura 1.
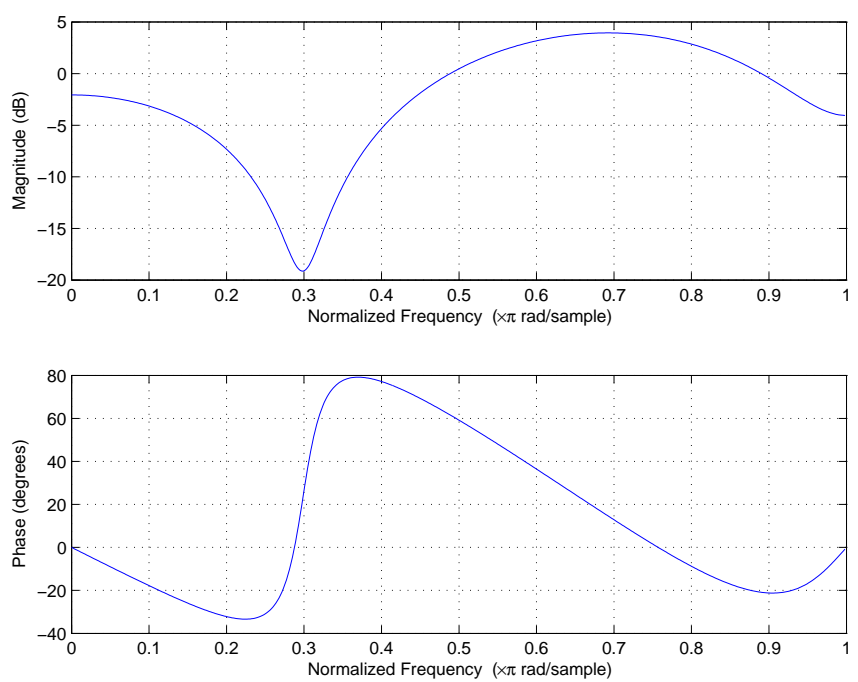

Fig. 1. Resposta em Freqüência do Canal de Ordem 5

O segundo tipo de canal é fixo no tempo, porém, aleatório sendo modelado como um filtro FIR com $L=6$ taps, cujos coeficientes são do tipo

$$
q_{l}=p_{l} \alpha_{l} ; \quad l=1,2 \ldots, L
$$

onde $\alpha_{l}$ é uma variável aleatória gaussiana complexa de média nula e $\mathbb{E}\left[\left|\alpha_{l}\right|^{2}\right]=1$. Os valores de $\alpha_{l}$ são sorteados no início de cada experimento e mantidos fixos ao longo de cada um destes. Os pesos $p_{l}$ satisfazem a $\sum_{l=1}^{L}\left|p_{l}\right|^{2}=1$. Os pesos utilizados são $p_{1}=0.74, p_{2}=-0.42, p_{3}=0.083, p_{4}=0.49$, $p_{5}=-0.12, p_{6}=0.01$, ou seja, neste canal os pesos são os coeficientes do canal tipo 1 .

A ambigüidade de fase inerente da estimação cega é eliminada adotando o primeiro coeficiente do vetor do canal como uma referência. Em todas as simulações adotamos equalização zero-forcing. O cenário considerado é: $K=16$ usuários ativos, número de subportadoras igual ao ganho de processamento $M=N=32$ e os códigos de espalhamento utilizados são sequiências curtas de Walsh-Hadamard. Os resultados ilustrados são a média de 100 experimentos independentes, cada um com até 2500 blocos de símbolos transmitidos.

Nas Figuras 2 a 5 estão ilustradas as curvas de desempenho do erro médio quadrático para o canal do $m$-ésimo usuário de interesse versus o número de blocos de símbolos transmitidos $N_{B}$. Em cada figura utilizou-se, respectivamente, um valor para a Razão Sinal Ruído: $10 \mathrm{~dB}, 15 \mathrm{~dB}, 20 \mathrm{~dB}, 25 \mathrm{~dB}$. Observa-se que em ambientes com alta razão sinal ruído, mesmo com pequenos valores da potência $p$, o desempenho de Erro Médio Quadrático do estimador baseado no método 
das potências aproxima-se bastante da curva de desempenho do estimador 'svd' (padrão) e em tais ambientes a potência $p=2$ é suficiente para a aproximação.

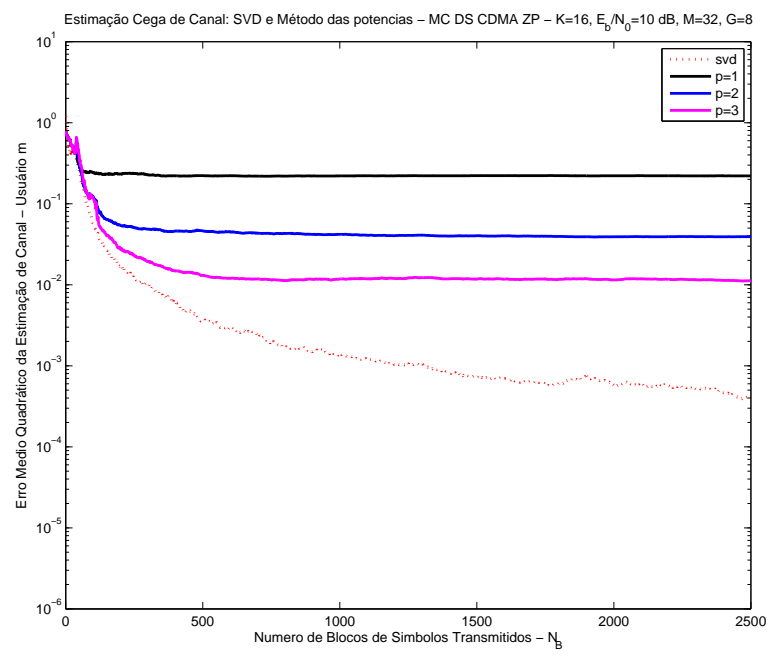

Fig. 2. Desempenho de Erro Médio Quadrático versus Número de Blocos de Símbolos Transmitidos $N_{B}$ - MC DS CDMA ZP - Canal Fixo-aleatório $E_{b} / N_{0}=10 \mathrm{~dB}$

Note que em ambientes de baixa Razão Sinal Ruído $E_{b} / N_{0}=10 \mathrm{~dB} E_{b} / N_{0}=15 \mathrm{~dB}$, Razão Sinal Ruído moderada $E_{b} / N_{0}=20 \mathrm{~dB}$ e Razão Sinal Ruído alta $E_{b} / N_{0}=25 \mathrm{~dB}$ a curva de desempenho do estimador obtido com aproximação em potência $p=3$ consegue um desempenho cada vez mais próximo ao desempenho do estimador 'svd' padrão.

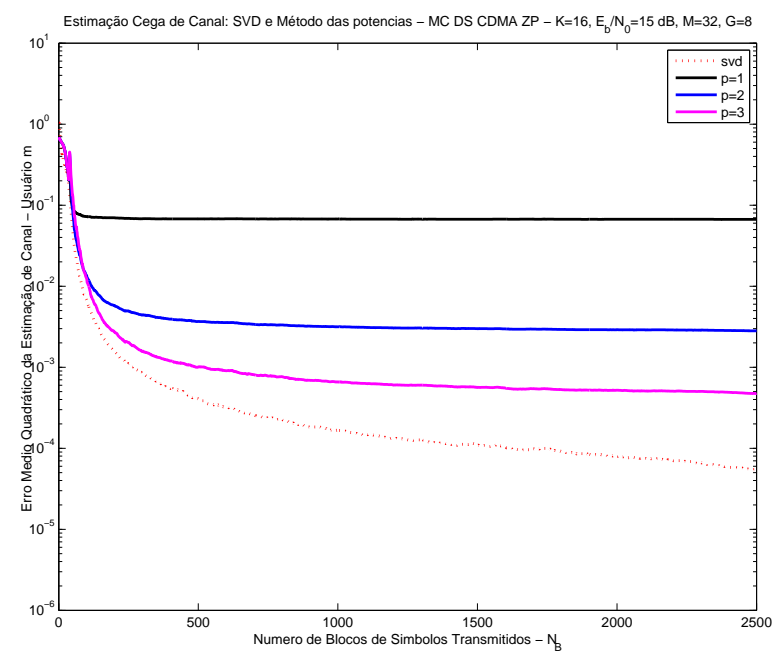

Fig. 3. Desempenho de Erro Médio Quadrático versus Número de Blocos de Símbolos Transmitidos $N_{B}$ - MC DS CDMA ZP - Canal Fixo-aleatório $E_{b} / N_{0}=15 \mathrm{~dB}$

Na maioria dos testes realizados para Razão Sinal Ruído moderada-alta, $E_{b} / N_{0}>20 d B$, os estimadores encontram desempenhos satisfatórios com $p=2$. Mais ainda, em ambientes de alta Razão Sinal Ruído, o estimador proposto com $p=1$ permite resultados aceitáveis. Nos experimentos para avaliar o desempenho de Erro Médio Quadrático os resultados obtidos com ambos os canais fixo e fixo-aleatório foram bastante semelhantes, por isso, optamos por ilustrar apenas as curvas de desempenho obtidas com o canal fixo-aleatório.

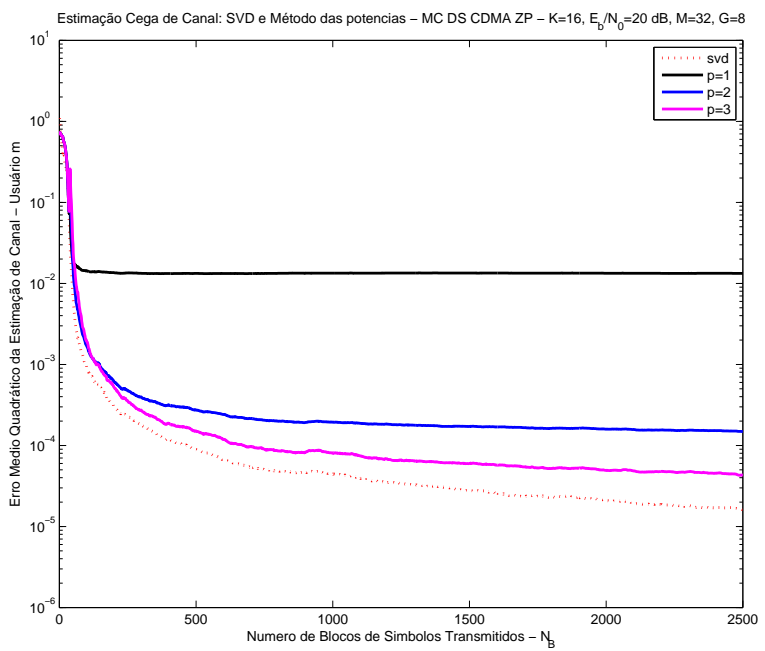

Fig. 4. Desempenho de Erro Médio Quadrático versus Número de Blocos de Simbolos Transmitidos $N_{B}$ - MC DS CDMA ZP - Canal Fixo-aleatório $E_{b} / N_{0}=20 \mathrm{~dB}$

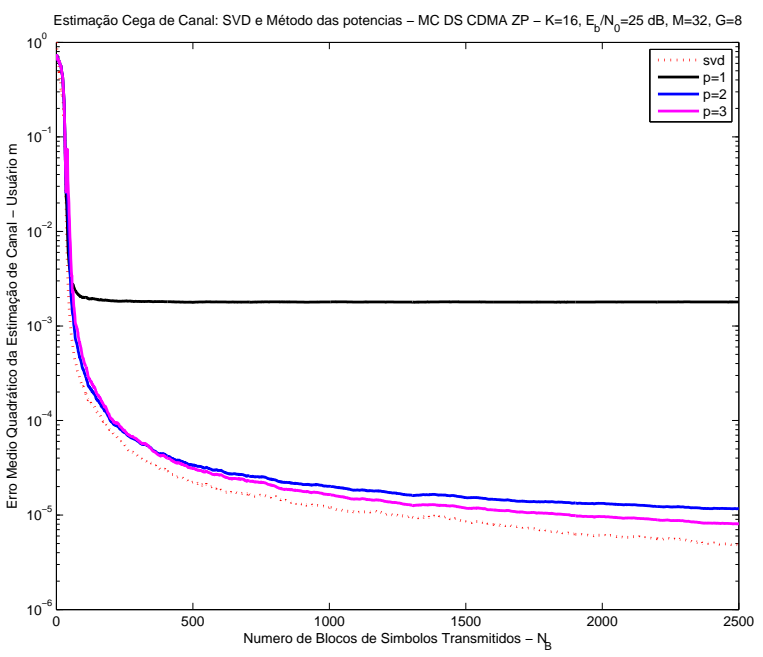

Fig. 5. Desempenho de Erro Médio Quadrático versus Número de Blocos de Símbolos Transmitidos $N_{B}$ - MC DS CDMA ZP - Canal Fixo-aleatório $E_{b} / N_{0}=25 \mathrm{~dB}$

Na Figura 6 estão ilustradas as curvas de desempenho da Taxa de Erro de Bits versus $E_{b} / N_{0}(\mathrm{~dB})$ para o mesmo cenário considerado para as figuras anteriores $(K=16$, $M=32, G=8$ ) mas em canal fixo. Pode ser confirmado pela distribuição das curvas que é suficiente considerar a potência $p=3$ para aproximar o produto do subespaço do ruído. Mais ainda, para razão sinal ruído, da ordem de $18 \mathrm{~dB}$, o estimador com aproximação por $p=2$ encontra desempenho bastante próximo ao desempenho do estimador 'svd' padrão, esse atinge nível de BER de $10^{-3}$ em $18 \mathrm{~dB}$ e os estimadores 
com potência $p=2$ e $p=3$, praticamente, atingem o mesmo patamar em $18.5 \mathrm{~dB}$.

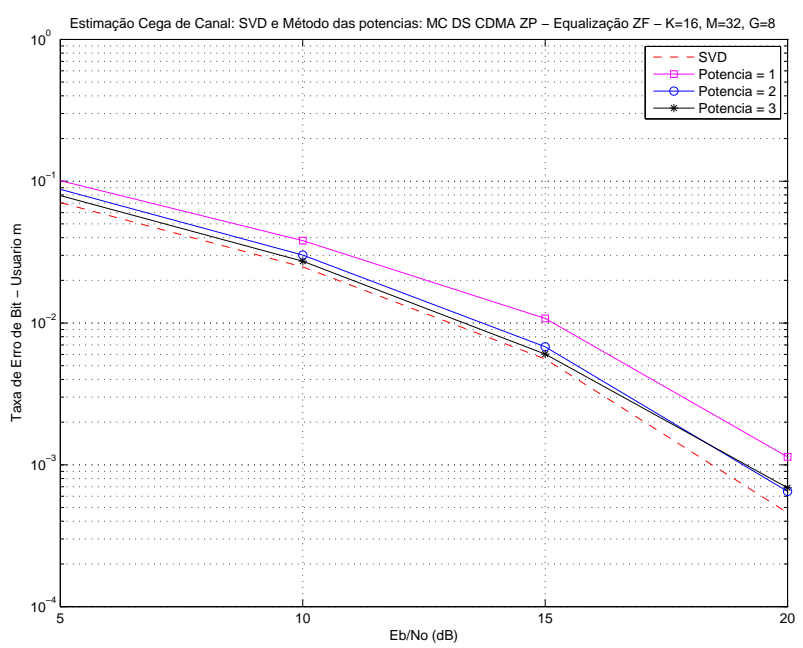

Fig. 6. Desempenho de Taxa de Erro de Bit (BER) versus Razão Sinal Ruído $E_{b} / N_{0}(\mathrm{~dB})$ - MC DS CDMA ZP - Canal Fixo

Na figura 7 utilizou-se o canal fixo-aleatório no mesmo cenário acima descrito, porém, o número de experimentos foi aumentado de 100 para 250 . Note que para a faixa de valores de $E_{b} / N_{0}$ considerados as curvas de desempenho do estimador com aproximação em $p=2$ e $p=3$ são, praticamente, iguais. Ademais, em ambientes com moderada razão sinal ruído, entre 15 a $20 \mathrm{~dB}$, para aproximar o produto do subespaço do ruído por uma matriz de potência pequenos valores de $p$ são suficientes $(p<3)$. $\mathrm{O}$ sistema usando o estimador obtido por aproximação em potência com $p=2$ é suficiente para obter desempenho muito próximo ao do sistema usando o estimador 'svd' padrão.

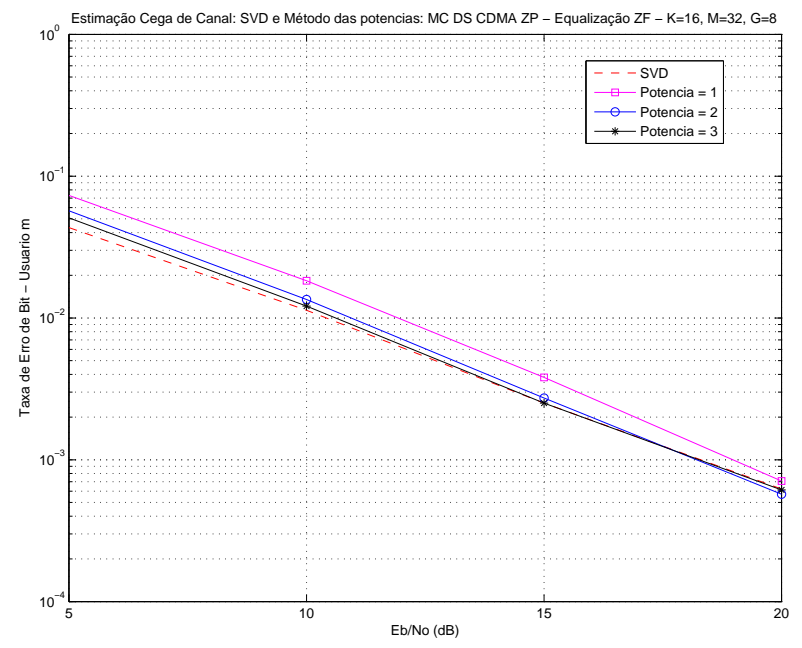

Fig. 7. Desempenho de Taxa de Erro de Bit (BER) versus Razão Sinal Ruído $E_{b} / N_{0}(\mathrm{~dB})$ - MC DS CDMA ZP - Canal Fixo-Aleatório

\section{Conclusões}

Este trabalho investigou o problema da estimação cega de canal baseada em métodos de identificação de subespaço em conjunto com minimização quadrática. A metodologia proposta foi aplicada ao sistema MC DS CDMA ZP e permitiu uma formulação alternativa de menor complexidade computacional. Na formulação para a obtenção da estimativa de canal foi usado o método das potências para aproximar o subespaço do ruído nas operações de decomposição por valor singular (SVD). Os resultados de desempenho, em ambientes com moderada Razão Sinal Ruído, mostraram que a estimativa baseada no método das potências permitiu um desempenho bastante próximo ao das abordagens de SVD 'tradicionais'. Para quaisquer dois valores de potência $p$, quando o número de blocos de símbolos aumenta, a maior potência prevalece, apresentando melhor concordância com o método de subespaço padrão, sendo que pequenos valores de $p$ são suficientes $(p \leq 3)$.

\section{REFERÊNCIAS}

[1] Z. Wang, G.B. Giannakis, Wireless Multicarrier Communications, IEEE Signal Processing Magazine, pp. 29-48, Maio, 2000.

[2] V. DaSilva and E. S. Sousa, Performance of orthogonal CDMA codes for quasi-synchronous communication systems, Proc. IEEE International Conference on Universal Personal Communications, ICUPC 93, pp. 995999, Outubro, 1993.

[3] S. Kondo e L. B. Milstein, On the use of multicarrier direct sequence spread spectrum systems, Proc. IEEE Military Communications Conference, MILCOM, pp. 52-56, Outubro, 1993.

[4] S. B. Weinstein e P. M, Ebert, Data Transmission by frequency division multiplexing using the discret fourier transform, IEEE Transactions 0n Communications Tech., COM-19(5), pp. 628-634, Outubro, 1971.

[5] M. K. Tsatsanis e Z. Xu, Performance Analysis of Minimum variance CDMA Receivers, IEEE Transactions Vehicular on Signal Processing,vol. 46, pp. 3014-3022, Novembro, 1998.

[6] Z. Xu e M. K. Tsatsanis, Blind Adaptive Algorithms for Minimum Variance CDMA Receivers, IEEE Transactions Vehicular on Communications,vol. 49, no. 1, pp.180-194, Janeiro, 2001.

[7] X. G. Doukopoulos e G. V. Moustakides, Blind Channel Estimation for Downlink CDMA Systems, Proc. IEEE International Conference on Communications (ICC), 2003.

[8] X. G. Doukopoulos e G. V. Moustakides, Power Techniques for Blind Adaptive Channel Estimation in CDMA Systems, Proc. IEEE Global Telecommunications Conference (GLOBECOM), 2003.

[9] Fabian David Backx, T. T. V. Vinhoza e Raimundo Sampaio Neto, Power Techniques for Blind Adaptive Channel Estimation in ZeroPadded OFDM Systems, 18th Annual IEEE International Symposium on Personal Indoor and mobile radio Communication PIMRC, 2007.

[10] B. Muquet, Z. Wang, G. B. Giannakis, M. de Courville e P.Duhamel, Cyclic Prefixing or Zero Padding for Wireless Multicarrier Transmissions?. IEEE Transactions On Communications, v. 12, n. 50, p. 21362148, 2003.

[11] G. H. Golub e C. F. Van Loan, Matrix Computation, 2a. edição. The John Hopkins University Press, 1990.

[12] X.G.Doukopoulos e G.V. Moustakides, Blind Channel estimation for DS-CDMA, INRIA - Relatório de Pesquisa no.4781, Março, pp. 1-20, 2003.

[13] H. Sari and G. Karam and I. Jeanclaude, Transmission Techniques for Digital Terrestrial TV Broadcasting, IEEE Communications Magazine, 0163-6804/95, pp. 100-109, 1995. 